\title{
Down regulation of surviving gene and up regulation of p53 gene expression by siRNA induces apoptosis in human hepatocellular carcinoma cell line HepG2
}

\author{
Yun-Hua Lu ${ }^{1,2,3}$, Cong Tang ${ }^{1,2}$, Wei Wang ${ }^{1,2}$, Tao $\mathrm{Xi}^{1,2^{*}}$ \\ ${ }^{1}$ Research Centre of Biotechnology, China Pharmaceutical University, NanJing, 210009, China. ${ }^{2}$ JiangSu Key Laboratory of Carcinogenesis and intervention, \\ China Pharmaceutical University. ${ }^{3}$ School of Chemistry \& Biotechnology, YiChun University, YiChun, JiangXi, 33600, China. *Correspondence should be \\ addressed to Yun-Hua Lu (lyh655@yahoo.com.cn)
}

Received April 16 $6^{\text {th }}, 2008$; revised October 31 $1^{\text {st }}, 2008$; accepted October $31^{\text {st }}, 2008$

\begin{abstract}
Survivin gene may be a good target for cancer gene therapy because it is over expressed in a variety of human tumors including human hepatocellular carcinoma but not in differentiated adult tissues. To explore the effects of the siRNA of survivin gene inducing apoptosis in human hepatocellular cancer cells, three siRNAs cpusiRNA1, cpusiRNA2 and cpusiRNA3 were designed and transferred into human hepatocellular carcinoma cell line HepG2 (HepG2) by lipofection. MTT test showed that the growth of HepG2 decreased when it was transfected with $25 \mathrm{nM}, 50 \mathrm{nM}, 100 \mathrm{nM}, 150 \mathrm{nM}, 200 \mathrm{nM}, 400 \mathrm{nM}$ siRNA respectively after 48 hours. And the change of mRNA and protein of survivin gene and p53 gene had been detected by RT-PCR and Western blot. Cells presented an increase in apoptosis index was assayed by flow cytometry. Small interfering RNA can exert a knockdown of survivin gene expression and up regulation of p53 gene to induce apoptosis and to inhibit the growth of HepG2.
\end{abstract}

Keywords: RNAi, Survivin gene, p53 gene, Apoptosis, HepG2

\section{INTRODUCTION}

Hepatocellular carcinoma (HCC) was the most pernicious cancer with a high mortality rate. Despite the improvement of surgical techniques and other conditions, the prognosis of HCC remained poor. Therefore, there is a need for development of new therapy way to improve the survival rate for potential use in HCC. Many studies have shown that the survivin gene was a new member of inhibitors of the apoptosis protein (IAP) family, which had been implicated in both control of cell division and inhibition of apoptosis. It was selectively overexpressed in the majority of human tumor types including liver, lung, breast, colon, hepatocellular, oesophageal, pancreatic, bladder, uterine and ovarian cancers, largecell nonHodgkin's lymphomas, leukaemias, neuroblastoma, brain tumors, pheochromocytoma, soft tissue sarcomas, melanomas and other skin cancer, but not in adult differentiated tissues with the exception of thymus and genital gland, and was associated with the aggressiveness of diseases and unfavorable outcomes[1,2,3,4,5]. Ling $\mathrm{X}$ et al reported that induction of survivin by taxol in MCF-7 cells was an early event and was independent of taxol-mediated G2/M arrest, thus suggesting a role for survivin in taxol resistance not only during mitosis but outside of the mitotic checkpoint as well[6]. On the basis of these findings survivin had been proposed as an attractive target for new anticancer interventions. Several preclinical studies had demonstrated that downregulation of survivin expression, accomplished through the use of small interfering RNA to increase the apoptotic rate and to reduce tumor growth potential. It could specifically and efficiently degrade mRNA, resulting in post uctranscriptional gene silencing (PTGS) $[7,8,9,10,11,12,13,14$, $15]$, which was a natural mechanism in organisms underlying the resistance to virus invasion and inhibition of transposon mobility. Its blocking action on gene expression had been successfully observed in rat and human cells cultured in vitro, and the knockdown of genes in cells had been achieved [11,15].A study[16] had shown that 21-25 nt small interference RNA (siRNA) could mediate specific gene silencing in mammal cells. Being effective and highly specific, RNAi probably becomed a new technique in knocking gene down and plays an important role in gene therapy of diseases. Three small interfering RNAs were designed according to the sequence of survivin gene and they were transferred into human hepatocellular carcinoma cell line HepG2 by lipofection to observe survivin and p53 gene expression changes and their effects on cell apoptosis and growth, 
which laid a foundation for further studies on the functions of survivin gene and genetic therapy involved in HCC.

\section{MATERIALS AND METHODS}

\subsection{Main Reagents}

Trizol reagent and M-MLV were purchased from Gibco BRL (Carlsbad, CA). Taq DNA RNA-Mate kit was bought from Genepharma (Shanghai, China). Polyclonal rabbit anti-human polymerase, dNTPs and DNA Marker were obtained from Takara (Dalian City, China). survivin, p53 and $\beta$-Actin antibody were purchased from WuHan Boster Co. (WuHan City, China).

\subsection{Small Interfering RNA Design}

CpusiRNA1 sense: 5'-ACUGCAGAGAAAGAGCC dTdT-3', cpusiRNA1 anti-sense: 5'-GGCUCUUUCUCU GUCCAGUTT-3'; cpusiRNA2 sense: 5'-GAAUUUGAG GAAACUGCGAdTdT-3', cpusiRNA2 anti-sense: 5'-UC GCAGUUUCCUAACUUACTT-3'; cpusiRNA3 sense: 5'-AGCAUUCGUCCGGUUGCGCdTdT-3', cpusiRNA3 anti-sense: 5'-GCGCAACCGGACGAAUGCUTT-3'; Negative control cpusiRNA4 sense: 5'-UUCUCCGAAC GUGUCACGUdTdT-3', Negative control cpusiRNA4 anti-sense: 5'-ACGUGACACGUUCGGAGAATT-3'. Each sense chain of siRNA oligonucleotides was added two thymidine residues (dTdT) at the 3 ' ends. After these siRNAs having been designed, they were compared with sequence in the human EST (expressed sequence tag) database to confirm that no other genes were targeted, and then they were sent to Shanghai Genepharma Co., Ltd (Shanghai city in China) to be synthesized.

\subsection{Primers of Survivin Gene Design}

After primers of survivin gene (forward primer P1: 5'CGACGTTGCCCCCTGCCTG3', reverse primer P2: 5'AAGGAAAGCGCAACCGGACGA3'), p53 gene (forward primer P3: 5'AGCGATGGTCTGGCCCCTCC3', reverse primer P4: 5' GCGCCGGTCTCTCCAGGA3') and GAPDH gene (forward primer P5: 5'-ATTCAACGG CACAGTCAAGG-3', forward primer P6: 5'-GCAGAA GGGGCGGAGATGA-3') having been designed, they were sent to Shanghai Sangon Biological Co. (China) to be synthesized.

\subsection{Cell Culture}

Human hepatocellular carcinoma cell line HepG2 was maintained in our laboratory. And it was grown in RPMI-1640 supplemented with $100 \mathrm{~mL} / \mathrm{L}$ fetal bovine serum (FBS) and incubated in a humidified incubator containing $50 \mathrm{~mL} / \mathrm{L} \mathrm{CO}_{2}$ at $37^{\circ} \mathrm{C}$.

\subsection{Transfection}

Twenty-four hours before transfection, cells were trpsinized, diluted in fresh media without antibiotics and transferred to 96-well and 6-well plates, These cells grown to a confluency of $50 \%-60 \%$ were transfected with $0,25 \mathrm{nmol} / \mathrm{L}, 50 \mathrm{nmol} / \mathrm{L}, 100 \mathrm{nmol} / \mathrm{L}, 200 \mathrm{nmol} / \mathrm{L}$ and $400 \mathrm{nmol} / \mathrm{L}$ of siRNA cpusiRNA1, cpusiRNA2 and cpusiRNA3 per well using RNAi-Mate media according to manufacturer's recommendations.

\subsection{MTT Assay}

HepG2 cell $\left(5 \times 10^{3}\right)$ was placed into every well of 96well plate with $180 \mu \mathrm{L}$ culture medium RPMI-1640 containing $10 \%$ NBS. After the cell having been incubated at $37^{\circ} \mathrm{C}$ for 24 hours, $20 \mu \mathrm{L}$ siRNA cpusiRNA1, cpusiRNA2 and cpusiRNA3 with increasing concentrations $0,25 \mathrm{nmol} / \mathrm{L}, 50 \mathrm{nmol} / \mathrm{L}, 100 \mathrm{nmol} / \mathrm{L}, 200 \mathrm{nmol} / \mathrm{L}$ and $400 \mathrm{nM}$ was added to every well respectively. When the cell had been incubated at $37^{\circ} \mathrm{C}$ for $44 \mathrm{~h}$, cell proliferation was assessed by MTT, After the cell having been incubated for $4 \mathrm{~h}$, the reaction was stopped by the addition of $150 \mu \mathrm{L}$ DMSO, After $30 \mathrm{~min}$ incubation and shaking, the absorbency of the samples was determined at $490 \mathrm{~nm}\left(\mathrm{~A}_{490}\right)$.

\subsection{RT-PCR}

Total RNA was extracted from HepG2 cell using Trizol reagent (Gibco BRL) according to the manufacturer's instructions. Complementary DNA (cDNA) was generated from total RNA using M-MLV. PCR of cDNA was performed in a final volume of $50 \mu \mathrm{L}$ containing $4 \mu \mathrm{L}$ of $4 \times$ dNTPs, 2 units of Taq DNA polymerase, and 20 $\mathrm{mmol} / \mathrm{L}$ of each primer. The samples were amplified 35 cycles at 94 for $30 \mathrm{~s}$, at 58 for $30 \mathrm{~s}$, and at 72 for $50 \mathrm{~s}$, and finally at 72 for $10 \mathrm{~min}$. Amplification of human GAPDH served as a control for a sample loading and integrity. the length of amplified fragments of survivin gene P53 and GAPDH gene were $250 \mathrm{bp}, 306 \mathrm{bp}$ and $213 \mathrm{bp}$.

\subsection{Western Blot}

The cells were washed twice with cold PBS and were lysed in radioimmunoprecitation assay buffer [50 $\mathrm{mmol} / \mathrm{L}$ of Tris (pH 7.4), $150 \mathrm{mmol} / \mathrm{L} \mathrm{NaCl}, 1 \%$ Triton $\mathrm{X}-100,1 \%$ deoxycholic phenylmethyl-sulfonyl fluoride, $1 \mu \mathrm{g} / \mathrm{ml}$ of aprotinin, and $1 \mathrm{mmol} / \mathrm{L}$ of DTT] for $10 \mathrm{~min}$ and scraped. The extracts were centrifuged at $4000 \mathrm{~g}$ at $4^{\circ} \mathrm{C}$ for $15 \mathrm{~min}$. Protein $(100 \mu \mathrm{g})$ were resolved by sodium dodecyl sulfatepolyacry-lamide gel electrophoresis (SDS-PAGE) and transferred to nitrocellulose membranes (Sigma Co., Ltd in USA). After blocking with 5\% skim milk in Tris- $\mathrm{HCl}(\mathrm{pH} 7.5)$ at room temperature for $2 \mathrm{~h}$, the nitrocellulose membranes were reacted for $2 \mathrm{~h}$ with specific antibodies in the same blocking solution. After extensive washing with Tris- $\mathrm{HCl}$ containing $0.05 \%$ Tween 20, the membrances were reacted with rabbit anti-human polyclonal antibody for Survivin, p53 and $\beta$-Actin protein detection.

\subsection{Flow Cytometric Analysis}

Cell cycle distributions were determined by measuring the cellular DNA content using flow cytometry (BD Biosciences Clontech, Palo Alto, CA). Cells were washed with PBS twice, fixed with $700 \mathrm{~mL} / \mathrm{L}$ ethanol for $20 \mathrm{~min}$ and stored at $4{ }^{\circ} \mathrm{C}$ overnight, then washed with PBS twice, and stained with $100 \mu \mathrm{L}$ of $50 \mathrm{mg} / \mathrm{L}$ PI 

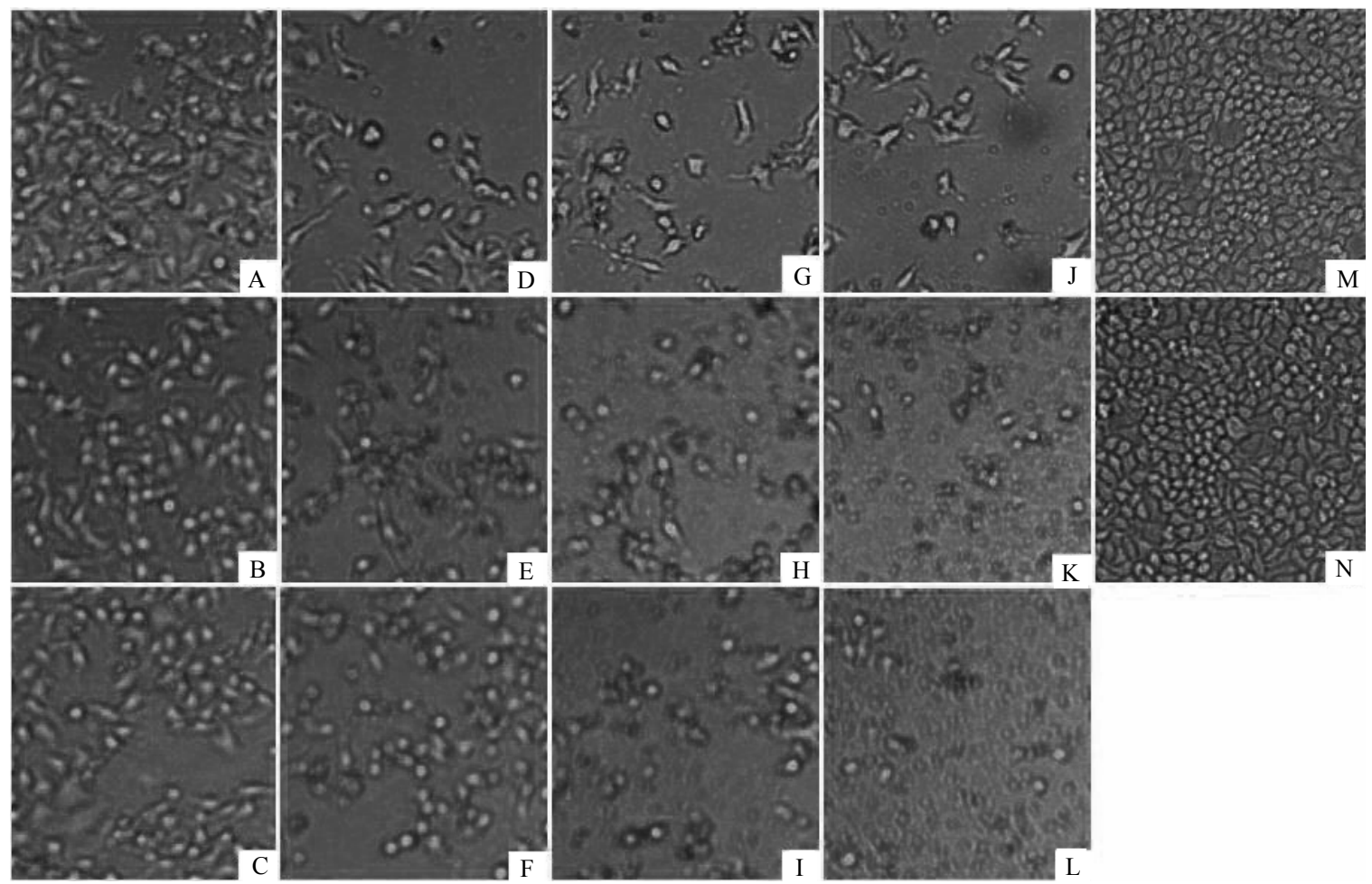

Figure 1. HepG2 cells transfected with siRNA. A-C HepG2 cells transfected with 50 nM nM of siRNA cpusiRNA1, cpusiRNA2 and cpusiRNA3 after 48 hours. D-F HepG2 cells transfected with 100 nM of siRNA cpusiRNA1, cpusiRNA2 and cpusiRNA3 after 48 hours. G-I HepG2 cells transfected with 200 nM of siRNA cpusiRNA1, cpusiRNA2 and cpusiRNA3 after 48 hours. J-L HepG2 cells transfected with 400 nM of siRNA cpusiRNA1, cpusiRNA2 and cpusiRNA3 after 48 hours. M HepG2 cells transfected with 200 nM of siRNA cpusiRNA4 after 48 hours. N Untransfected HepG2 cells.

at $4{ }^{\circ} \mathrm{C}$ for $30 \mathrm{~min}$. Apoptotic cells were assayed using the Elite ESP flow cytometry at $488 \mathrm{~nm}$, and data were analyzed with the WinMID2.9 software.

\subsection{Statistical Analysis}

Data were expressed as mean \pm SD. Statistical significance was determined by the Students' t-test. $\mathrm{P}<0.05$ was considered statistically significant.

\section{RESULTS}

\subsection{Change of Cellular Morphology}

After the cells were transfected with $0,50 \mathrm{nM}, 100 \mathrm{nM}$, 200nM and 400nM of siRNA cpusiRNA1, cpusiRNA2 and cpusiRNA3 respectively for $48 \mathrm{~h}$, The cellular morphology of HepG2 cells had changed greatly, such as the volume of cell changing small, the cellular morphology becoming irregularity, cell shrinking, and nucleus pycnosis. But the cellular morphology of control cells was normal (Figure 1).

\subsection{Cellular Proliferation}

The number of the cells became small when the cells were tranfected with $0,25 \mathrm{nM}, 50 \mathrm{nM}, 100 \mathrm{nM}, 200 \mathrm{nM}$ and $400 \mathrm{nM}$ of siRNA cpusiRNA1, cpusiRNA2 and cpusiRNA3 respectively after 48 hours, and the inhibi- tion effect to the HepG2 fade up in evidence. But the negative control cpusiRNA4 have no evident effect on the growth of HepG2 cells. The inhibition rate was determined by MTT assay as Table 1. And tumor curative Cisplatin was used as positive control to inhibit the growth of HepG2 cells $10 \mu \mathrm{M}, 20 \mu \mathrm{M}, 40 \mu \mathrm{M}$, $80 \mu \mathrm{M}$ and $160 \mu \mathrm{M}$. The inhibition rate was determined by MTT assay as Table 1 and 2 . The $\mathrm{IC}_{50}$ of the cpusiRNA2 and cpusiRNA3 to the HepG2 cells is $88.1 \mathrm{nM}, 89.2 \mathrm{~nm}$, while the $\mathrm{IC}_{50}$ of Cisplatin is $32.1 \mu \mathrm{M}$ which is about 365 times higher than that of cpusiRNA2 and cpusiRNA3.

Table 1. Effects of siRNA and Cisplatin on the growth of HepG2 cells determined by MTT assay $(n=6)$, (mean \pm SD)

\begin{tabular}{cccccc}
\hline siRNA & $\mathbf{0}$ & $\mathbf{2 5 n M}$ & $\mathbf{5 0 n M}$ & $\mathbf{1 0 0 n M}$ & $\mathbf{2 0 0 n M}$ \\
\hline cpusiRNA1(\%) & 0 & $17.2 \pm 2.6$ & $36.6 \pm 4.2$ & $50.2 \pm 3.7$ & $66.3 \pm 3.8$ \\
cpusiRNA2(\%) & 0 & $16.8 \pm 2.5$ & $33.9 \pm 4.2$ & $54.5 \pm 3.7$ & $75.3 \pm 5.2$ \\
cpusiRNA3(\%) & 0 & $10.5 \pm 3.4$ & $20.3 \pm 3.9$ & $41.7 \pm 3.6$ & $61.5 \pm 4.2$ \\
Cisplatin & 0 & $10 \mu \mathrm{M}$ & $20 \mu \mathrm{M}$ & $40 \mu \mathrm{M}$ & $80 \mu \mathrm{M}$ \\
Cisplatin(\%) & 0 & $16.1 \pm 3.8$ & $42.4 \pm 4.3$ & $83.1 \pm 4.1$ & $92.7 \pm 3.9$ \\
\hline
\end{tabular}



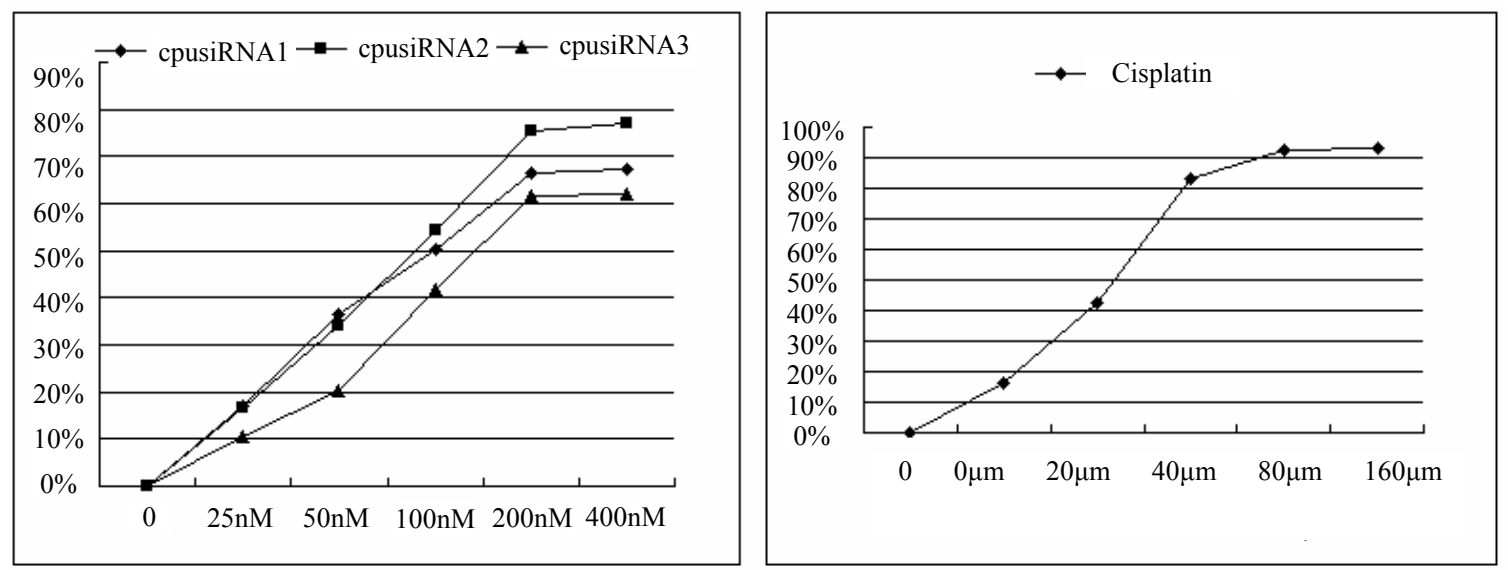

Table 2. The curve of the effects of siRNA and Cisplatin on the growth of HepG2 cells

\subsection{Change of the Survivin and p53 mRNA}

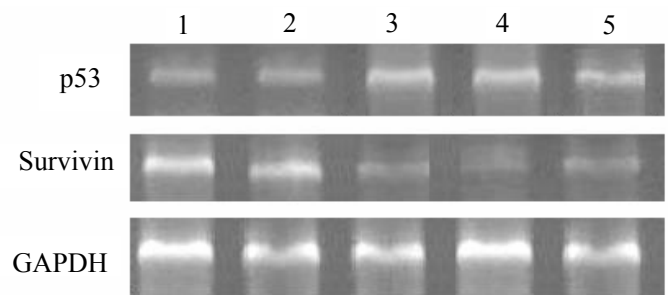

Figure 2. RT-PCR show the change of survivin and p53 mRMA. 1 HepG2 cells transfected with $200 \mathrm{nM}$ of siRNA cpusiRNA4 after 48 hours; 2 Untransfected HepG2 cells; 3. 4. 5. HepG2 cells transfected with $100 \mathrm{nM}$ of siRNA cpusiRNA1, cpusiRNA2 and cpusiRNA3 after 48 hours

Four-eight hours after the transfection of survivin siRNA, the semi-quantitative reverse transcription polymerase chain reaction amplified $250 \mathrm{bp}$ fragment of survivin gene, $306 \mathrm{bp}$ fragment of p53 gene and $213 \mathrm{bp}$ fragment of GAPDH gene as Figure 2. The result suggested not only that the mRNA of survivin gene declined, but also that the mRNA of p53 gene increased when the cells were transfected with $100 \mathrm{nM}$ of siRNA cpusiRNA1, cpusiRNA2 and cpusiRNA3 at $48 \mathrm{~h}$ in HepG2 cells (Figure 2).

\subsection{Change of the Survivin and p53 Protein}

After four-eight hours treatment of siRNA, the Western blot showed that the amount of survivin protein reduced with suppression of the survivin mRNA, and that the expression of p53 gene up-regulated because of increasing of p53 mRNA when HepG2 cells were transfected with $100 \mathrm{nM}$ of siRNA after 48 hours as Figure 3.

\subsection{Flow Cytometry Analysis}

After the HepG2 cells were transfected with 100nM of siRNA cpusiRNA4, cpusiRNA1, cpusiRNA2 and

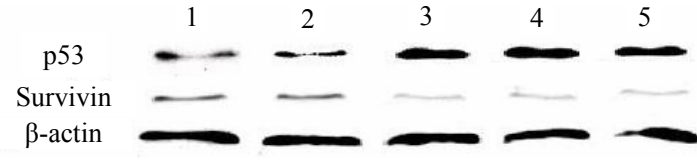

Figure 3. Western blot showed the change of survivin and p53 protein. 1. HepG2 cells transfected with $100 \mathrm{nM}$ of siRNA cpusiRNA4 after 48 hours; 2 . Untransfected HepG2 cells; 3. 4. 5. HepG2 cells transfected with 100 nM of siRNA cpusiRNA1 cpusiRNA2 and cpusiRNA3 after 48 hours

cpusiRNA3 respectively, flow cytometry analysis of their cell cycle displayed that the cells transfected with $100 \mathrm{nM}$ of siRNA cpusiRNA1, cpusiRNA2 and cpusiRNA3 respectively with a marked reduction in $\mathrm{G} 2 / \mathrm{M}$ phase by $8.3 \%, 16.2 \%$ and $21.5 \%$, the apoptosis index was as high as $13.4 \%, 26.6 \%$ and $32.1 \%$. And the control groups transfected with $200 \mathrm{nM}$ of siRNA cpusiRNA4 is almost the same as the untransfected HepG2 (Figure 4).

\section{DISCUSSION}

Tumorigenesis is a multiple factor course. The high expressed inhibited-apoptosis genes in tumor cells can suppress apoptosis and make tumor cells avoid to be cleared and identified by the immunity system. Moreover, the inhibited apoptosis genes can also play a role during the course of drug resistance of tumor cells. Survivin provides a new research direction to cancer therapy for itscharacters of high conservation and only expression in tumor cells Small interfering RNA technology possesses a high ability to specifically silence particular genes. Therefore, it can be used as a powerful tool in researches on the functions of genes and genetic therapy for carcinoma. Attention has been paid to RNAi in the field of researches on gene functions.

In our study, the result of MTT assay demonstrated that the chemically synthesized siRNA cpusiRNA1, 

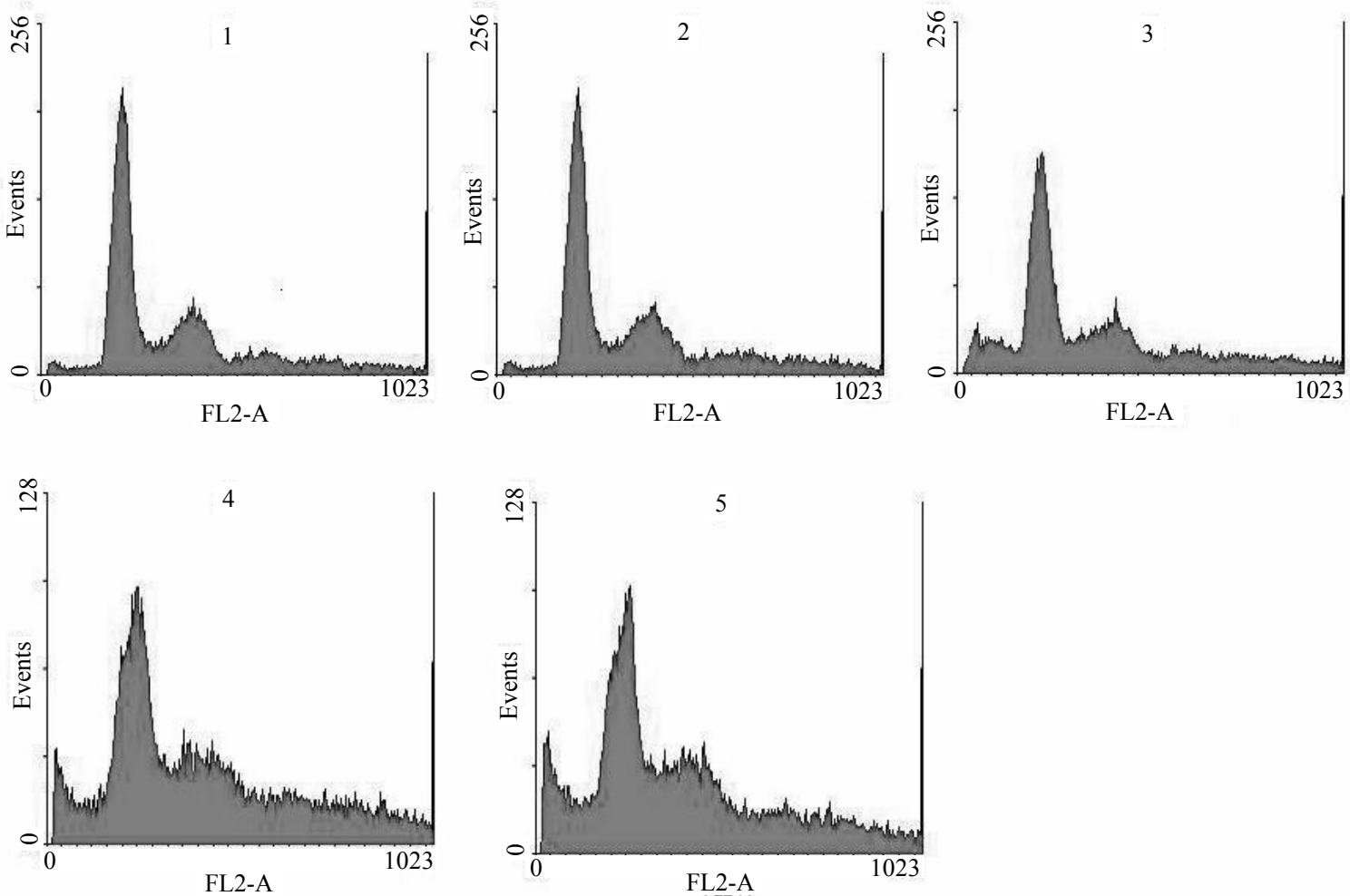

Figuer 4. Result of cell cycle detected by flow cytometry. 1 HepG2 cells transfected with $200 \mathrm{nM}$ of siRNA cpusiRNA4 after 48 hours; 2 Untransfected HepG2 cells; 3 HepG2 cells transfected with 200 nM of siRNA cpusiRNA1 after 48 hours; 4 HepG2 cells transfected with $200 \mathrm{nM}$ of siRNA cpusiRNA2 after 48 hours; 5 HepG2 cells transfected with $200 \mathrm{nM}$ of siRNA cpusiRNA3 after 48 hours

cpusiRNA2 and cpusiRNA3 can effectively inhibit thegrowth of the HepG2 cells. Their $\mathrm{IC}_{50}$ to the HepG2 cells is about $1 / 187,1 / 232$ and $1 / 127$ less than that of Cisplatin. The result of RT-PCR demonstrated not only that survivin gene was exerted a knockdown, but also that the mRNA of p53 gene increased at the level of transcription when the cells were transfected with $100 \mathrm{nM}$ of siRNA cpusiRNA1, cpusiRNA2 and cpusiRNA3 at $48 \mathrm{~h}$ in HepG2 cells. The result of Western blot demonstrated that survivin gene was exerted a knockdown and p53 gene was up-regulation in HepG2 cells at the level of protein. Flow cytometry analysis revealed that the HepG2 cells transfected with the siRNA cpusiRNA1, cpusiRNA2 and cpusiRNA3 presented an obvious AP peak, a marked reduction in $\mathrm{G} 2 / \mathrm{M}$ phase and an increase in apoptosis index compared to the control groups. We also observed that HepG2 cells transfected with the siRNA cpusiRNA1, cpusiRNA2 and cpusiRNA3 grew slowly, as compared with the control groups. The above mentioned findings confirm that chemically synthesized siRNAs can specifically block survivin gene expression, induce cell apoptosis, and inhibit the growth of carcinoma cells.

The results of our study not only confirm that the inhibitory activity of survivin gene on the growth of human hepatocellular carcinoma cell line HepG2 could be realized by inducing cell apoptosis, but also confirm that the survivin gene interfering on the growth of human hepatocellular carcinoma cell line HepG2 could upregulate p53 gene expression to inhibit the growth of HepG2 cells. Besides, RNAi alone could block survivin gene expression to induce a remarkable increase in cell apoptosis. This unique effect of survivin provides new evidence for its antiapoptotic effects on HepG2. In summary, survivin gene can be regarded as a very good target gene in genetic therapy for carcinomas. RNAi of survivin gene is a promising approach in treating carcinomas.

\section{REFERENCES}

[1] H. Caldas, L. E. Honsey and R. A. Altura. (2005) Survivin a novel Survivin splice variant expressed in malignancies. Mol Cancer, 4(1): 11-23.

[2] W. Salz, D. Eisenberg and J. Plescia. (2005) A survivin gene signature predicts aggressive tumor behavior. Cancer Res, 65: 3531-3534.

[3] T. Liu and D. Grossman. (2004) Rapid induction of mitochondria events and caspase-independent apoptosis in Survivin-targeted melanoma cells. Oncogene, 23 (1): 39-48.

[4] M. Stevenson. (2004) Therapeutic potential of RNA interference. N Engl J Med, 351 (17): 1772-1777.

[5] D. C. Altieri; (2003) Survivin versatile modulation of cell division and apoptosis in cancer. Oncogene, 22: 8581-8589.

[6] X. Ling, R. J. Bernacki, M. G. Brattain and F. Li. (2004) Induction of survivin expression by taxol (paclitaxel) is an early event, which is independent of taxol-mediated G2/M arrest. J Biol Chem; 
279: (5)196-203.

[7] C. D. Novina and P. A. Sharp. (2004) The RNAi revolution. Nature, 430(6996): 161-164.

[8] P. D. Zamore. (2002) Ancient pathways p rogrammed by small RNAs. Science, 296 (5571): 1265-1269.

[9] G. J. Hannon. (2002) RNA interference. Nature, 418: 2442-2511.

[10] M. Pennati, M. Binda, M. De Cesare, G. Pratesi, M. Folini, L. Citti, M. G. Daidone, F. Zunino and N. Zaffaroni. (2004) Ribozyme-mediated down-regulation of survivin expression sensitizes human melanoma cells to topotecan in vitro and in vivo. Carcinogenesis; 25: 1129-1136.

[11] M. Izquierdo. (2005) Short interfering RNAs as a tool for cancer gene therapy. Cancer Gene Ther, 12: 217-227.

[12] M. Kappler, M. Bache, F. Bartel, M. Kotzsch, M. Panian, P. Wurl, K. Blumke, H. Schmidt, A. Meye and H. Taubert. (2004) Knockdown of survivin expression by small interfering RNA reduces the clonogenic survival of human sarcoma cell lines independently of p53. Cancer Gene Ther, 11: 186-193.

[13] M .Kappler, H. Taubert, F. Bartel, K. Blumke, M. Panian, H. Schmidt, J. Dunst and M. Bache. (2005) Radiosensitization, after a combinedtreatment of survivin siRNA and irradiation, is correlated with the activation of caspases 3 and 7 in a wtp53 sarcoma cell line, but not in a mt-p53 sarcoma cell line. Oncol Rep, 13: $167-172$.

[14] M. Chawla-Sarkar, S. I. Bae, F. J. Reu, B. S. Jacobs, D. J. Lindner and E. C. Borden. (2004) Downregulation of Bcl-2, FLIP or IAPs (XIAP and survivin) by siRNAs sensitizes resistant melanoma cells to Apo2L/TRAIL-induced apoptosis. Cell Death Differ, 11: 915-923.

[15] S. Coma, V. Noe, C. Lavarino, J. Adan, M. Rivas, M. Lopez-Matas, R. Pagan, F. Mitjans, S. Vilaro, J. Piulats and C. J. Ciudad; (2004) Use of siRNAs and antisense oligonucleo -tides against survivin RNA to inhibit steps leading to tumor angiogenesis. Oligonucleotides, 14: 100-113.

[16] S. M. Elbashir, W. Lendeckel and T. Tuschl. (2002) RNA interference is mediated by 21-and 22-nucleotide RNAs. Genes Dev, 15: $188-200$.

[17] Corresponding author; Prof. Xi Tao, Research Centre of Biotechnology, China Pharmaceutical University, NanJing, 210009, China. 